\title{
Optimization and Design of Hammerheads and Fenders on Scrap Metal Shredders Based on Improved Genetic Algorithm
}

\author{
He Tian $\left(\mathbb{D}\right.$, Guoqiang Wang, Kangkang Sun, Zeren Chen, Chuliang Yan $\mathbb{D}^{D}$, and Da Cui ${ }^{\circ}$ \\ School of Mechanical and Aerospace Engineering, Jilin University, Changchun, China \\ Correspondence should be addressed to Da Cui; cuidajlu@outlook.com
}

Received 15 July 2021; Revised 17 November 2021; Accepted 19 November 2021; Published 24 December 2021

Academic Editor: Thanh-Phong Dao

Copyright ( $) 2021 \mathrm{He}$ Tian et al. This is an open access article distributed under the Creative Commons Attribution License, which permits unrestricted use, distribution, and reproduction in any medium, provided the original work is properly cited.

\begin{abstract}
Dynamic unbalance force is an important factor affecting the service life of scrap metal shredders (SMSs) as the product of mass error. Due to the complexity of hammerheads arrangement, it is difficult to take all the parts of the hammerhead into account in the traditional methods. A novel optimization algorithm combining genetic algorithm and simulated annealing algorithm is proposed to improve the dynamic balance of scrap metal shredders. The optimization of hammerheads and fenders on SMS in this paper is considered as a multiple traveling salesman problem (MTSP), which is a kind of NP-hard problem. To solve this problem, an improved genetic algorithm (IGA) combined with the global optimization characteristics of genetic algorithm (GA) and the local optimal solution of simulated annealing algorithm (SA) is proposed in this paper, which adopts SA in the process of selecting subpopulations. The optimization results show that the resultant force of the shredder central shaft by using IGA is less than the traditional metaheuristic algorithm, which greatly improves the dynamic balance of the SMS. Validated via ADAMS simulation, the results are in good agreement with the theoretical optimization analysis.
\end{abstract}

\section{Introduction}

Due to the existence of machining errors and the difference of process parameters, the machining process of hammerheads will cause a mass error. However, for the SMS, when the hammerheads of different masses are installed on the rotor and the rotor rotates, the dynamic unbalance force will be produced, which may reduce the service life of the rotor. The arrangement of hammerheads will affect the result of the dynamic unbalance force, so the arrangement of hammerheads is important to the design of SMS.

At present, the traditional method of hammerhead arrangement is to ensure rough equality of the transverse and longitudinal mass of hammerheads or to adopt the method of a large mass of hammerheads in the middle of the rotor and a small mass of hammerheads on both sides of the rotor. These two methods can effectively reduce the dynamic unbalance of the rotor, but the result is not optimal due to the fact that the hammerhead is divided into three different types, and the arrangement of hammerheads is more complex, so it is very difficult to meet the requirements.
This paper focuses on the optimization of hammerhead arrangement with PFY2026 which consists of 39 hammerheads, 6 side fenders, and 21 common fenders. A kind of arrangement is designed to minimize the dynamic balance of SMS. This problem belongs to the multiple traveling salesman problem (MTSP) which is an extension problem of the famous traveling salesman problem (TSP) and belongs to the NP-hard. MTSP is more complex than TSP because it aims to solve a set of Hamilton loops where $m(m>1)$ is formed in $n(n>m)$ and the salesman does not walk repeatedly.

Many problems are used to model MTSP, including the many-objective optimization problem for an unmanned aerial vehicle [1], the optimization of vehicle crashworthiness [2], aircraft fuselage stiffeners [3], the shape optimization of a vehicle clutch lever [4], mission planning [5], and vehicle scheduling [6, 7]. Because of the complexity of MTSP combinations, many researchers try to relax MTSP to standard TSP and solve it by using an exact algorithm, but the results are not satisfactory [7]. The fuzzy-logic-based Taguchi method is an effective approach for optimizing the 
multiple quality characteristics of compliant mechanisms [8-10]. For the MTSP problem, various optimization algorithms, such as ant colony algorithm (ACO), genetic algorithm (GA), simulated annealing algorithm (SA), artificial bee colony algorithm $(\mathrm{ABC})$, artificial neural network (ANN), and particle swarm optimization (PSO), are all studying the optimization and improvement of the problem. In [11], a new ACO followed by the MAX-MIN Ant System is proposed to solve the MTSP. Compared with GA, this method demonstrates competitive performance on some benchmark instances. To optimize the arrangement of hammerheads and fenders, Wang et al. [12] proposed an ant colony optimization algorithm improved by a multidimensional matrix and random number $\mathrm{q} 0$ and separating the first loop from other loops. Simulation results show that this method can significantly reduce the dynamic unbalance force.

ACO is specially proposed for TSP and performs well. ACO has many advantages, such as good robustness, parallel distributed computing, and easy combination with other heuristic methods. However, because the convergence speed of ACO is slow, it is easy to stagnate and to be trapped in a local optimal solution. Compared with ACO, GA is a global optimization algorithm [13]. This characteristic avoids many disadvantages of traditional local search techniques in difficult search spaces. In particular, hybridization is a very powerful mechanism for introducing new genetic material and maintaining genetic diversity, but with decisive attributes, good parents also produce good performance or superior offspring [14-16]. Carter et al. [17] proposed a new GA chromosome and its related operators for MTSP, comparing its theoretical properties and computational properties with previous studies. Their computational tests show that the two-part chromosome coding method leads to a smaller search space and, in many cases, leads to better solutions than previous techniques. Most importantly, this work minimizes the number of redundant candidate solutions in the search space. To improve the GA, Kwak et al. [18] implemented a new selection method and a novel crossover operation in the genetic algorithm. The capacities of the proposed strategies are verified through various nonlinear/ nonconvex functions and engineering optimization problems. Cho et al. [19] used GA and ANN to optimize the structure of truck radial tires, which can effectively improve the durability of the tires.

Meng et al. present a comprehensive work on the application of ten popular and recent metaheuristic algorithms to five engineering problems [20]. The efficiency of nine metaheuristic algorithms is evaluated on eight mechanical design problems using solution quality and convergence analysis [21].

GA is extensible and easy to be mixed with other technologies. Therefore, based on the defects of GA in the TSP problem, it can be combined with other optimization algorithms, which may achieve unexpected results. Simulated annealing (SA) is a general probabilistic search technique that simulates physical processes of heating and controlled cooling [22]. SA attempts to replace the current state with random movement at each step. New states are acceptable, and their probabilities depend both on the temperature difference between the corresponding function values and on the differences between them. Therefore, SA is capable of obtaining more optimal solutions [23]. Lee et al. [23] proposed a new method combining a genetic algorithm and a truncated Monte Carlo simulation to reduce optimal cost. Wang et al. [24] proposed a novel genetic simulated annealing (GSA), which is a combination of GA and SA, to optimize the machining parameters for milling operations. Furthermore, to solve the optimal reactive power programming problem, Jwo et al. [25] proposed a global optimization technique and hybrid simulated annealing/ genetic algorithm (HSAGA) combining GA and SA. In the actual operation of the reactor, this method shows improved performance. In order to overcome the slow convergence speed and poor global search ability of quantum-inspired evolutionary algorithms, Deng et al. proposed a variety of improved methods for different algorithms. In [26], an improved quantum-inspired cooperative coevolution algorithm is proposed. And an enhanced MSIQDE algorithm based on mixing multiple strategies is described in [27]. They combined the quantum computing characteristics of quantum evolutionary algorithm (QEA) and the divide-andconquer idea of cooperative coevolution evolutionary algorithm (CCEA) to come up with an improved differential evolution in [28].

In this paper, an improved genetic simulated annealing algorithm (IGA) is proposed, in which the triple traveling salesman problem is set up in parallel by real number coding to optimize the dynamic balance of the arrangement of hammerheads and fenders on scrap metal shredders (SMSs). The hybrid of GA and SA is an innovative attempt. It employs the superior performance of the SA algorithm to solve the local optimal solution and improves the convergence of GA in optimization. In the operation of GA, selection, crossover, and mutation share the same chromosome. And before the selection, the three variables are split, and after the mutation is finished, the three variables are reconstituted into the same chromosome. In the SA, parallel operation is also used, which performs well at the local optimal solution. Finally, comparing with the optimization results of ACO which are the same as Wang [12], the resultant force of the shredder rotor center shaft is reduced by $80.64 \%$, and the dynamic balance of the shredder rotor is greatly improved.

The paper is arranged as follows. In Section 2, the dynamic balance theory and the mathematical model of the SMS are presented. Section 3 details the basic structure and parameter setting of the proposed IGA. In Section 4, the optimization results of the IGA are compared with those of other algorithms. In Section 5, the results are analyzed and verified by ADAMS. Finally, conclusions are drawn in Section 6.

\section{Mathematical Model of SMS}

2.1. Dynamic Balance Design Theory. According to the dynamic balance theory, there will be a dynamic unbalance of the rotating rotor due to machining errors and installation 
errors. In order to achieve the dynamic balance of the rotating rotor, mass can be added or reduced to counteract the dynamic unbalance. When a well-balanced rotor is installed, the required assembly tolerances allow radial displacement, resulting in an unbalanced state. Thus, although the dynamic unbalance of the rotor cannot be completely eliminated, its influence can be minimized as much as possible.

2.2. SMS Parameters. As shown in Figure 1, the SMS consists of 39 hammerheads, 6 side fenders, and 21 common fenders. The working radius is $2000 \mathrm{~mm}$ and the working length is $2790 \mathrm{~mm}$. The rotational speed of the rotor is $595 \mathrm{r} / \mathrm{min}$. The actual mass of hammerheads and fenders is shown in Tables 1 and 2 .

The arrangement form of hammerheads and fenders is shown in Table 3. The hammerheads and fenders are intersected, in which the fenders protect the hammerhead. According to experience, the arrangement of hammerheads also meets the following requirements:

(1) The mass of the hammerheads and fenders on the same disk is equal or similar

(2) Hammerheads and fenders cross in heavy and light form

(3) The hammerheads arranged in the axis direction are symmetrically arranged relative to both sides of the centerline

\subsection{Dynamic Balance Model of Shredder}

2.3.1. Global and Local Coordinates. This paper defines $o_{i}$ $-x_{i} y_{i} z_{i}(i=1, \ldots, 69)$ as the coordinate system of the shredder rotor system, as shown in Figure 2 and it is also the global coordinate system of the SMS, the local coordinate system of the hammerhead plate, and the local coordinate system of the hammerheads.

The component of a position vector in coordinate system $o_{i}-x_{i} y_{i} z_{i}$ is defined as follows:

$$
r_{i}=\left\{\begin{array}{c}
x_{i} \\
y_{i} \\
z_{i}
\end{array}\right\},
$$

where $r_{i}$ is the coordinates of the centroids of the $i$-th rotor and it can be converted to coordinate system $o_{i-1}-x_{i-1} y_{i-1} z_{i-1}$ via

$$
r_{i-1}=d_{i-1}+A_{i-1, i} r_{i},
$$

where $d_{i-1}$ represents the position vector of coordinate system $o_{i}-x_{i} y_{i} z_{i}$ relative to coordinate system $o_{i-1}-x_{i-1} y_{i-1} z_{i-1}$ and $A_{i-1, i}$ represents the rotation matrix of coordinate system $o_{i}-x_{i} y_{i} z_{i} \quad o_{i}-x_{i} y_{i} z_{i}$ relative to $o_{i-1}-x_{i-1} y_{i-1} z_{i-1}$.

For example, when analyzing the local coordinate system $o_{4}-x_{4} y_{4} z_{4}$ of the hammerhead relative to the coordinate system $o_{3}-x_{3} y_{3} z_{3}$, it is considered that the local coordinate system is as follows:

$$
r_{4}=d_{4}+A_{4,3} r_{3},
$$

where, if the rotor of SMS reaches dynamic balance, the displacement vector $d_{4}$ can be regarded as 0 , and the coordinate system $o_{4}-x_{4} y_{4} z_{4}$ is only one angle $\theta=\pi / 3$ of rotation relative to the coordinate system $o_{3}-x_{3} y_{3} z_{3}$, namely,

$$
\begin{aligned}
d_{4} & =0 \\
A_{4,3} & =\left[\begin{array}{ccc}
\cos \theta & -\sin \theta & 0 \\
\sin \theta & \cos \theta & 0 \\
0 & 0 & 1
\end{array}\right] .
\end{aligned}
$$

The central shaft is a flexible body in practice, and there is deformation in the central shaft. However, the effect of the distribution of the hammerheads on the central shaft is small, so it is assumed that the central shaft is a rigid body.

2.3.2. Modeling. According to the principle of dynamic balance, two equivalent planes are set at both ends of the rotor to calculate the equivalent resultant force of the rotor, as shown in Figure 3. The mass of the hammerheads will affect the product of the mass radius, thus affecting the dynamic balance of the rotor.

Planes 1-11 are the 11 rows of the rotors, and plane I and plane II are the bearing central surfaces perpendicular to the central shaft at both ends of the central shaft. In the working process of SMS, both the hammerheads and the common fenders are in the state of swinging up and down. Even if they are hit by minerals, they will only swing back and forth at a very small angle, which is ignored here. Therefore, it can be assumed that the central shaft axis is in line with the center of mass of the hammers and the fenders. The radius of rotation of each hammer and fender is equal to the distance between the central shaft axis and their center of mass.

The SMS has a total of 11 disks. Each disk can be designed as a plane because, in the actual production process, there is a gap between the disk and the rotor connection, which leads to the shaking of hammerheads and fenders in the working process, but the angle is very small. This situation is ignored here. Therefore, it can be assumed that the rotation radius of the hammerheads and fenders is equal to the vertical distance from the centerline of the axis to the center of mass.

In the process of rotor rotation, it is assumed that there will be no movement back and forth between the hammerhead shaft and the relative movement between the hammerhead and the hammerhead shaft, so the distance between plane I and plane II and the distance between the two sides of the hammerhead are all known. The centrifugal force $\boldsymbol{F}_{i}=m r_{i} \omega^{2}, i=1, \ldots, 66$, the radius of the center of mass of the hammerhead is $r_{C}$, and the radius of the center of mass of the common fender is $r_{\mathrm{F}}$. When the rotor rotates at an angular velocity of $\omega$, the centrifugal inertia force is $F_{1} \sim F_{66}$, respectively, which forms a space force system. The conditions for dynamic balancing of the rotor are as follows: 


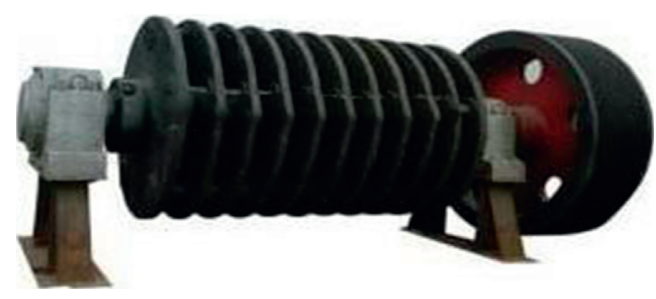

(a)

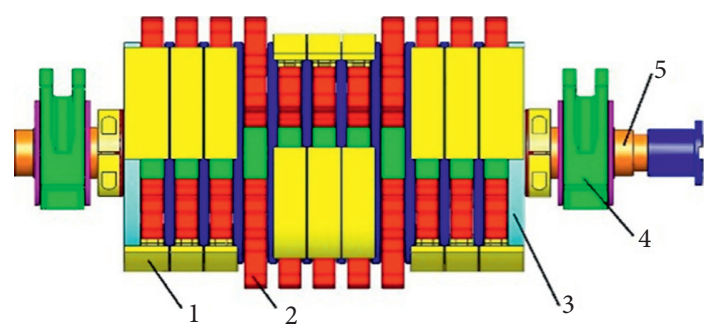

1 Common fender, 2 Hammerhead, 3 Hammerhead shaft, 4 Bearing, 5 Central shaft

(b)

Figure 1: The model of SMS. (a) Physical model of SMS (PFY2026); (b) three-dimensional model of SMS.

TABLE 1: Mass of hammerheads.

\begin{tabular}{lllllllll}
\hline Type & \multicolumn{7}{c}{ Mass $(\mathrm{kg})$} \\
\hline \multirow{2}{*}{ Heavy } & 135.0 & 134.3 & 134.2 & 134.1 & 134.1 & 134.1 & 134.1 & 134.0 \\
& 134.0 & 134.0 & 134.0 & 134.0 & & & & \\
\multirow{2}{*}{ Middle } & 133.4 & 133.4 & 133.2 & 133.2 & 133.2 & 133.2 & 133.0 & 133.0 \\
& 133.0 & 133.0 & 132.4 & 132.3 & 132.3 & 132.3 & 132.3 & 132.3 \\
\multirow{2}{*}{ Light } & 132.2 & 132.2 & 132.2 & 132.1 & 132.0 & 132.0 & 132.0 & 132.0 \\
& 131.3 & 131.3 & 130.2 & 130.0 & & & & \\
\hline
\end{tabular}

TABLE 2: Mass of fenders.

\begin{tabular}{lccccccc}
\hline \multicolumn{1}{c}{ Type } & & \multicolumn{5}{c}{ Mass (kg) } \\
\hline \multirow{3}{*}{ Common } & Heavy & 225.1 & 225.0 & 224.4 & 224.4 & 224.2 & 224.0 \\
fenders & Middle & 223.8 & 223.8 & 223.3 & 223.3 & 223.2 & 223.2 \\
& & 223.1 & 223.1 & 222.5 & 222.3 & 222.1 & 221.4 \\
\multirow{3}{*}{ Side fenders } & Light & 221.0 & 220.2 & 220.1 & & & \\
& Heavy & 260.0 & 255.4 & 255.4 & & & \\
& Light & 255.3 & 255.3 & 255.2 & & \\
\hline
\end{tabular}

TABle 3: Arrange form of the hammerheads and the fenders.

\begin{tabular}{|c|c|c|c|c|c|c|c|c|c|c|c|}
\hline & 1 & 2 & 3 & 4 & 5 & 6 & 7 & 8 & 9 & 10 & 11 \\
\hline A & 0 & 0 & 0 & 0 & $\square$ & $\square$ & $\square$ & 0 & 0 & 0 & 0 \\
\hline B & $\triangle$ & $\square$ & $\square$ & 0 & 0 & 0 & 0 & 0 & $\square$ & $\square$ & $\triangle$ \\
\hline C & 0 & 0 & 0 & 0 & $\square$ & $\square$ & $\square$ & 0 & 0 & 0 & 0 \\
\hline D & $\triangle$ & $\square$ & $\square$ & 0 & 0 & 0 & 0 & 0 & $\square$ & $\square$ & $\triangle$ \\
\hline E & 0 & 0 & 0 & 0 & $\square$ & $\square$ & $\square$ & 0 & O & 0 & 0 \\
\hline $\mathrm{F}$ & $\triangle$ & $\square$ & $\square$ & 0 & 0 & 0 & 0 & 0 & $\square$ & $\square$ & $\triangle$ \\
\hline
\end{tabular}

Note. $\bigcirc, \square, \triangle$ represent the arrangement form hammerheads, common fenders, and side fenders, respectively.

$$
\begin{aligned}
\sum_{i=1}^{66} F_{i} & =0, \\
\sum_{i=1}^{66} M_{i} & =0,
\end{aligned}
$$

where $\boldsymbol{F}_{i}$ is the centrifugal and $\boldsymbol{M}_{i}$ is the torque of the $i$-th hammerhead acting on the central shaft, respectively. The spatial inertial force system $F_{1} \sim F_{66}$ generated by the eccentric mass of the hammerhead is decomposed to plane I, as shown in Figure 3. Marked as $m_{\mathrm{I} A C}$, representing the mass of hammerheads at all $\mathrm{A}$ positions of 1-11 planes, $m_{\mathrm{IAF}}$, representing the mass of the common fenders at all $\mathrm{A}$ positions of 1-11 planes decomposed to plane I, $m_{\mathrm{I} B C}, m_{\mathrm{I} B F}$, $m_{\mathrm{I} C C}, m_{\mathrm{ICF}}, m_{\mathrm{IDC}}, m_{\mathrm{IDF}}, \ldots, m_{\mathrm{I} F F}$. In the same way, the spatial inertial force generated by the mass of the hammerhead is decomposed to plane II, $m_{\mathrm{IIAC}}$, representing the mass of hammerheads in all positions A of 1-11 planes to plane II, $m_{\mathrm{IIAF}}$, representing the mass of the common fenders at all $\mathrm{A}$ positions of 1-11 planes that are decomposed to plane II, $m_{\mathrm{II} B C}, m_{\mathrm{II} B F}, \ldots, m_{\mathrm{II} F C}, m_{\mathrm{IIFF}}$.

For plane I, the total resultant force is as follows:

$$
\begin{aligned}
F_{\mathrm{I}} & =\sum_{i} m_{i} r_{i} \omega^{2} \\
& =\sum_{i} m_{i} r_{i} \omega^{2}, \quad i=1, \ldots, 66
\end{aligned}
$$

In the same way, for plane II, the total resultant force is as follows:

$$
\begin{aligned}
F_{\mathrm{II}} & =\sum_{i} m_{i} r_{i} \omega^{2} \\
& =\sum_{i} \frac{L_{\mathrm{II} i}}{L} m_{i} r_{i} \omega^{2}, \quad i=1, \ldots, 66 .
\end{aligned}
$$

Therefore, the total combined force of plate I and plate II is as follows:

$$
\begin{aligned}
F & =F_{\mathrm{I}}+F_{\mathrm{II}} \\
& =\sum_{i}\left(\frac{L_{\mathrm{I} i}}{L} m_{i} r_{i} \omega^{2}+\frac{L_{\mathrm{II} i}}{L} m_{i} r_{i} \omega^{2}\right), \quad(i=1, \ldots, 66) .
\end{aligned}
$$
be

The optimization objective function can be assumed to

$$
\operatorname{Min}(F) \text {, }
$$

where $L$ represents the distance between plane I and plane II and $L_{\mathrm{I} i}$ and $L_{\mathrm{II} i}$ are the distance between the $i$-th hammerhead to the plane I and plane II, respectively, $m_{i}$ and $\boldsymbol{r}_{i}$ are the mass and the barycenter of the $i$-th hammerhead, while $\omega$ is the rotor rotation speed. 


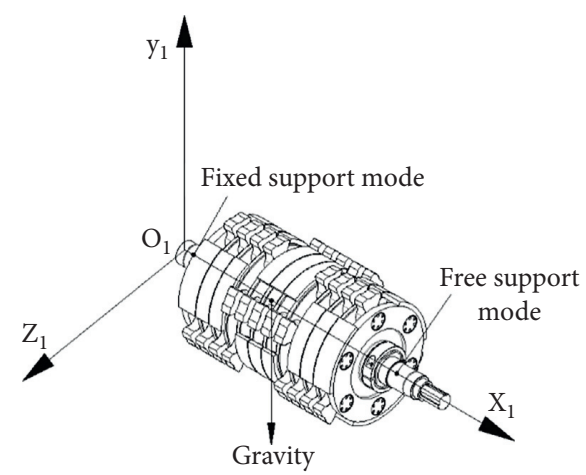

(a)

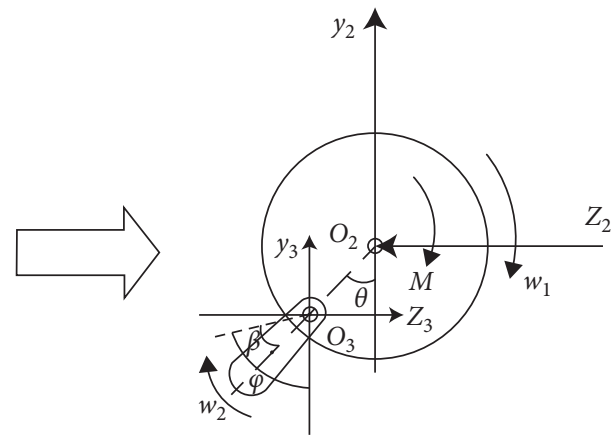

(b)

Figure 2: Coordinate system definition.
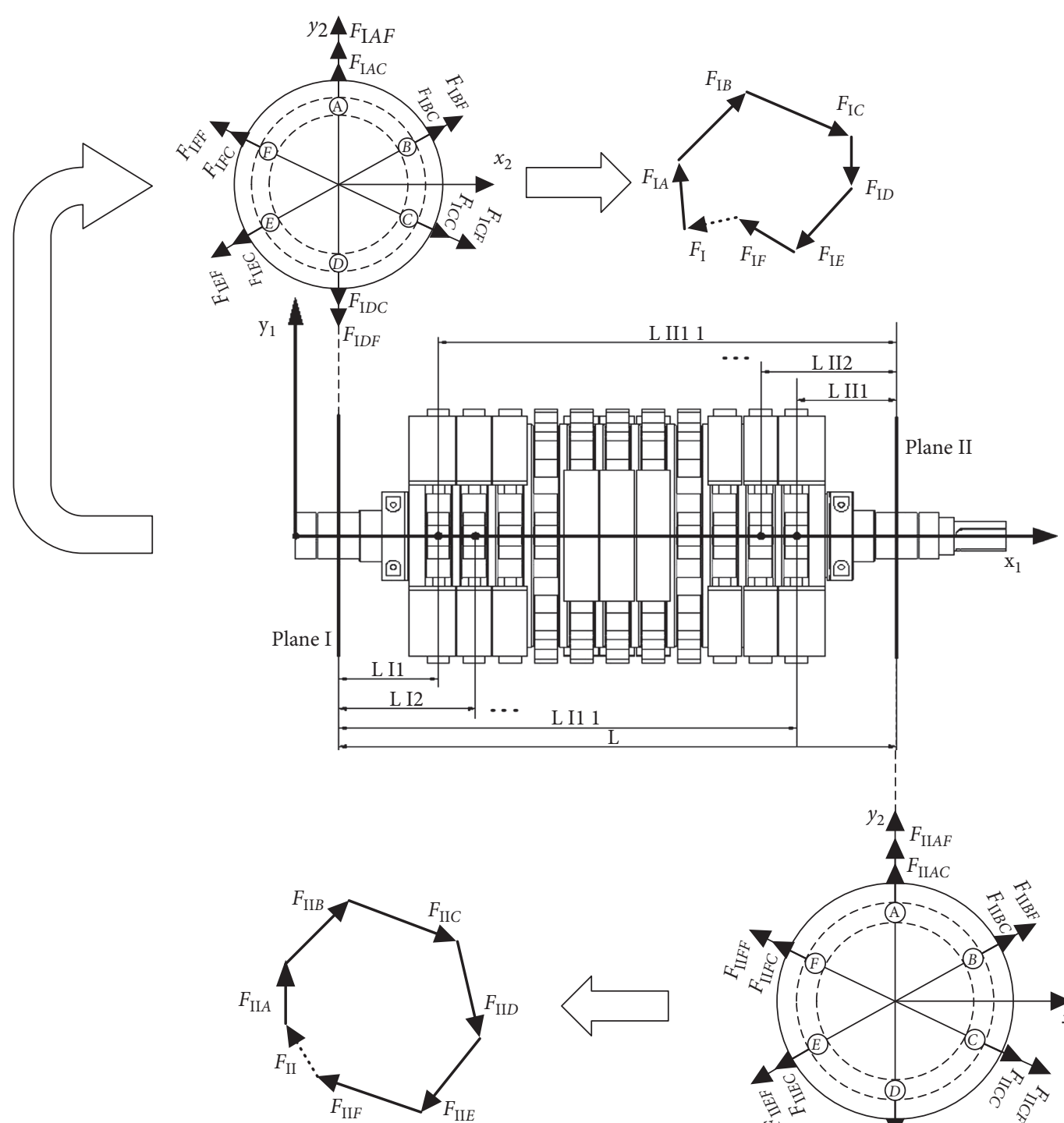
TABle 4: Arrangement of hammerheads and fenders of IGA and ACO (kg).

\begin{tabular}{lcccccccccccc}
\hline IGA & 1 & 2 & 3 & 4 & 5 & 6 & 7 & 8 & 9 & 10 & 11 \\
\hline A & 132.3 & 132.1 & 132.3 & 134.1 & 224.4 & 224.0 & 224.0 & 134.0 & 134.0 & 132.2 & 133.0 & 1736.4 \\
B & 255.2 & 220.2 & 225.1 & 134.0 & 132.4 & 133.0 & 132.2 & 133.0 & 224.0 & 222.1 & 255.4 & 2066.6 \\
C & 133.0 & 134.2 & 131.3 & 132.3 & 223.2 & 224.2 & 223.2 & 131.3 & 132.0 & 132.2 & 133.2 & 1730.1 \\
D & 255.4 & 222.5 & 223.1 & 134.3 & 133.2 & 133.2 & 134.1 & 135.0 & 221.0 & 223.2 & 260.0 & 2075 \\
E & 134.0 & 134.1 & 130.2 & 134.0 & 220.1 & 225.0 & 221.4 & 130.0 & 134.1 & 133.0 & 132.0 & 1727.9 \\
F & 255.3 & 222.3 & 223.1 & 134.0 & 132.0 & 134.0 & 132.0 & 133.0 & 224.4 & 223.3 & 255.3 & 2068.7 \\
Total & 1165.2 & 1065.4 & 1065.1 & 802.7 & 1065.3 & 1073.4 & 1066.9 & 796.3 & 1069.5 & 1066 & 1168.9 & 11404.7 \\
\hline ACO & 1 & 2 & 3 & 4 & 5 & 6 & 7 & 8 & 9 & 10 & 11 & Total \\
A & 135.0 & 132.2 & 134.1 & 132.0 & 224.2 & 220.1 & 223.1 & 133.0 & 132.3 & 131.3 & 134.1 & 1731.4 \\
B & 260.0 & 223.2 & 220.2 & 134.0 & 132.0 & 132.2 & 133.0 & 132.1 & 224.0 & 225.0 & 255.5 & 2071.2 \\
C & 133.0 & 133.0 & 132.3 & 130.0 & 224.4 & 225.1 & 223.2 & 130.2 & 134.0 & 133.2 & 133.2 & 1731.6 \\
D & 255.4 & 224.0 & 224.4 & 134.2 & 134.0 & 131.3 & 134.0 & 132.0 & 222.5 & 221.0 & 255.3 & 2068.1 \\
E & 132.3 & 134.0 & 134.1 & 134.0 & 222.3 & 223.2 & 221.4 & 132.0 & 133.0 & 134.1 & 134.0 & 1734.4 \\
F & 255.2 & 224.0 & 223.1 & 132.4 & 133.2 & 133.0 & 132.2 & 134.3 & 222.1 & 223.2 & 255.3 & 2067.9 \\
Total & 1170.9 & 1070.5 & 1068.1 & 796.6 & 1070.1 & 1064.9 & 1066.9 & 793.6 & 1067.9 & 1067.8 & 1167.3 & 11404.7 \\
\hline
\end{tabular}

The design variable is the arrangement of hammerheads which includes hammerheads, common fenders, and side fenders.

Constraints are given as follows:

(1) The arrangement of the three types of hammerheads is shown in Table 4 .

(2) The central shaft meets the stiffness conditions:

$$
\begin{gathered}
|f| \leq[f] \\
|\theta| \leq[\theta] .
\end{gathered}
$$

(3) The stiffness condition of the central shaft is as follows:

$$
\sigma_{\max }=\frac{|M|_{\max }}{W} \leq[\sigma]
$$

\section{Improved Genetic Algorithm (IGA)}

Genetic algorithm (GA) is widely used in the optimization field because of its extensive representation of feasible solutions, better global search performance, and inherent heuristic random search. GA approaches the optimal solution by increasing the fitness of individuals. The SA algorithm has strong global search ability and high search accuracy, but it also has some limitations: GA is easy to fall into the local optimal solution, while the accuracy and speed of SA are strongly dependent on the selection of the initial temperature and the process of cooling, and the convergence speed is very slow.

In this paper, an improved genetic algorithm (IGA) is proposed to optimize the balanced model of SMS with SA. The basic program block diagram process is shown in Figure 4. The algorithm consists of a GA stage and an SA stage, which are based on the genetic genes in biology.

In the first stage, GA calculates the optimal solution by selection, crossover, and mutation. In order to improve the GA falling into the local optimal solution, we introduce SA in the process of selecting subpopulations. SA jumps out of the local optimal solution trap through the adaptive temperature updating.

3.1. Crossover Strategy. GA uses single crossover and twopoint crossover as two basic genetic crossover operators mostly [29]. A single crossover is the most basic crossover operator proposed by Holland [30], and the concrete operation process is realized: a single crossover is randomly selected in a single string, genes are exchanged before and after the intersection, and then two new individuals are generated. In an earlier study, Spears et al. [31] found that because of increased genetic damage, two points, especially multiple points, encourage search space exploration. This helps prevent premature convergence of highly healthy individuals and makes search more robust. In this paper, a two-point crossover is adopted, and the crossover strategy is shown in Figure 5.

3.2. Mutation Strategy. The performance of GA depends on an efficient search operator to guide the system to global optimization. One problem besetting GA is premature convergence. In order to reduce or even avoid falling into a local optimal state, mutation operators provide a mechanism to explore new solutions and maintain population diversity [31]. Tang proposed an adaptive directed mutation (ADM) operator, which combines a local directed search strategy and an adaptive random search strategy to improve the genetic algorithm's ability to search for global optimal solutions and accelerate convergence [32].

There are many mutation operators such as random mutation operator (RMO) [33], polynomial mutation operator (PMO) [34], nonuniform mutation operator (NUMO) [35], and Gaussian mutation operator (GMO) [36-38]. A random mutation operator with a mutation rate of 0.01 is used in this paper. The parameter configuration of the IGA is shown in Table 5.

3.3. Selection Strategy Based on Simulated Annealing. The successful application of GA depends on the population size of the search space or the diversity of individual solutions. If 


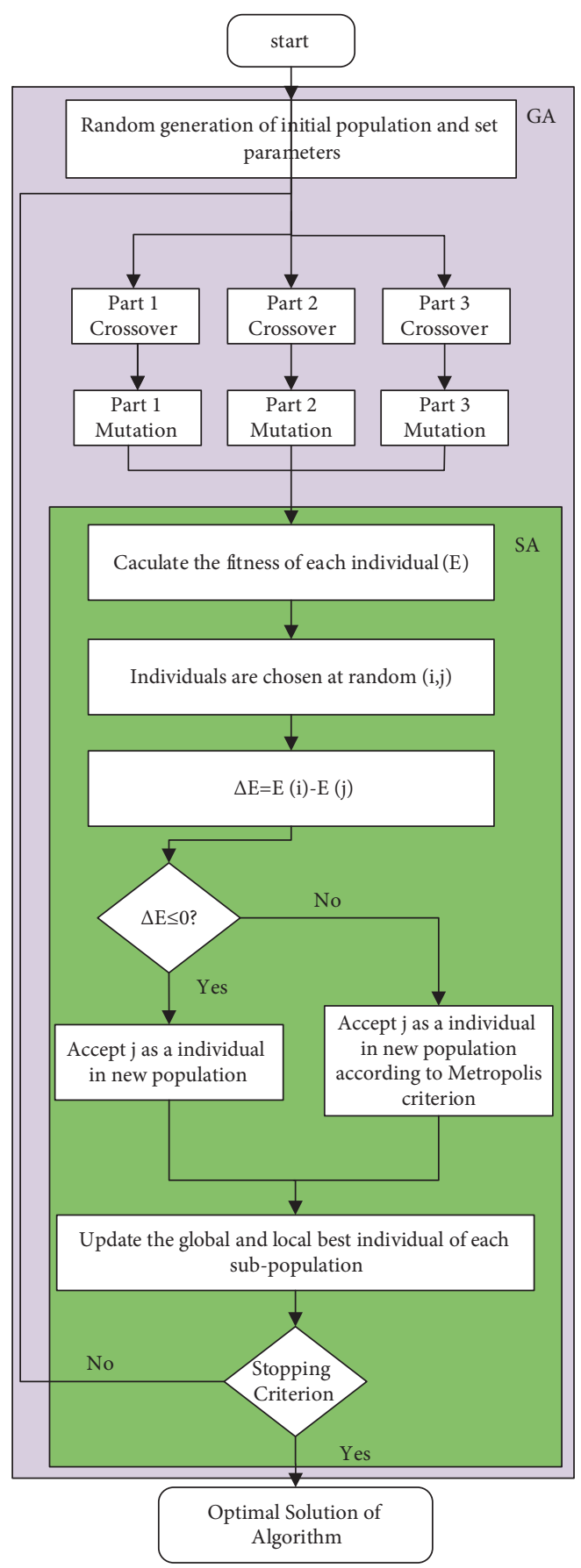

FIgURe 4: Block diagram of IGA.

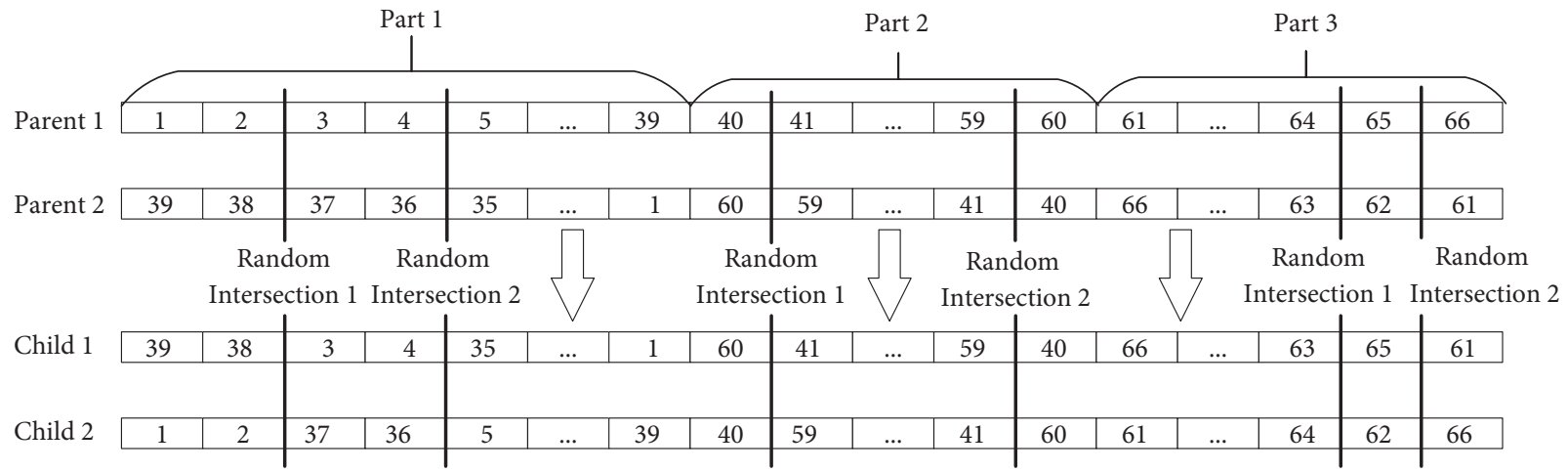

FIgURE 5: Two-point crossover strategy. 
TABle 5: Parameter configuration of IGA.

\begin{tabular}{lc}
\hline Parameter & Value \\
\hline popsize & 30 \\
$P_{c}$ & 0.85 \\
$P_{m}$ & 0.01 \\
Selection method in GA & Selection based on fitness \\
Mutation method & Swap \\
Replacement in GA & Steady-state GA \\
Replacement percentage & $1 / 3$ \\
$T_{0}$ & 97 \\
$T_{f}$ & 3 \\
$\alpha$ & 0.99 \\
Markov_length & 1000 \\
\hline
\end{tabular}

the diversity of GA cannot be maintained until it reaches the global optimum, it is difficult to find the global optimum, and sometimes it even leads to premature convergence to the local optimal. Although diversity is a major concern, it also leads to the deterioration of GA performance [39]. Many scholars have made many improvements based on GA, for example, combining simulated annealing algorithm (SA).

SA can also be directly applied to the MTSP problem. Because SA is a stochastic probability algorithm, the optimization consumes more time but shows more feasible results. Based on the above advantages, SA is applied to the process of solving the local optimal solution by MTSP. SA has the ability to jump out of the local optimization, and it is introduced into the IGA.

At present, the main selection strategies are the roulette selection method, random traversal sampling method, truncation selection method, and tournament selection method, based on previous studies. We introduce SA into the selection strategy to update the iterative population.

The SA starts from the initial setting temperature and generates the initial solution in the problem solution space and generates new solution through genetic crossover and mutation. By comparing the new solution with the old solution, the replacement is carried out according to the Metropolis criterion. SA accepts the optimal solution with probability 1 and the worst solution with a certain probability, which can effectively jump out of the local optimal solution.

The Metropolis criterion defines the probability of internal energy transfer from state $i$ to state $j$ at a certain temperature $T$ :

$$
p_{i j}^{T}= \begin{cases}1, & E(i) \leq E(j), \\ e^{-(E(i) \leq E(j) / K T)}=e^{-(\Delta E / K T)} & \text { others. }\end{cases}
$$

$E(i)$ and $E(j)$ are the internal energy in state $i$ and $j$, respectively; $\triangle E$ denotes the increment of internal energy; and $K$ is the Boltzmann constant. When $E(i) \leq E(j)$, the system will accept the new state with probability 1 ; on the contrary, the system will accept the bad state with a certain probability. The SA is used to improve the genetic operator to combine them. This probability is controlled by temperature $T$. When the temperature drops slowly enough, the optimization results will not easily jump out of the promising search area, which greatly enhances the local search ability of particles.

\section{Optimization Results of the Comparison}

In this section, we simulate IGA and compare it with several classical metaheuristic algorithms. ACO is based on the result of the previous paper [12]. The parameters include information heuristic factor $\alpha=1$; expectation heuristic factor $\beta=2$; and informational volatile factor $q 0=[0.7,0.9]$. Particle swarm optimization (PSO) is also a classical metaheuristic algorithm as a comparison in this paper. The main parameters of PSO include inertia weight $\omega=0.9$; cognitive acceleration coefficient $C 1=1$; and social acceleration coefficient $C 2=1$. The parameter configurations of GA and IGA are listed in Table 5. IGA optimizes the hammerheads in Tables 1-3. To illustrate the stability of IGA, the optimal results are solved by optimizing the theoretical results randomly in MATLAB. All the experiments corresponding to each algorithm are conducted 20 times independently. The numerical results obtained by various algorithms are presented in Table 6. Several statistics are recorded, such as the best, mean, worst, and standard deviation of the fitness values. In order to visualize and compare the convergence behavior, the history of the obtained best fitness value is plotted for each algorithm in Figure 6.

From Table 6, we can see that the IGA algorithm has higher stability. And compared with the classical metaheuristic algorithm, it can effectively escape the local trap and get the global optimal solution. By comparison in Figure 6, it is found that IGA has a faster convergence rate and the solution obtained is better.

\section{Simulation Verification and Discussion}

IGA optimizes the hammerheads in Tables 1-3. The parameter configurations of IGA are listed in Table 5. In Table 4, the arrangement of IGA and ACO take $F_{\min }=147.7 \mathrm{~N}$ and $762.94 \mathrm{~N}$, for example, respectively. The results show that the hammerheads and the fenders are intersected in the form of heavyweight, well balanced on the same radial plane, and similar axisymmetric mass. In IGA and ACO, the total mass of rows and columns are close, while the variation of column mass of IGA is less than that of ACO.

Table 4 shows that the results of IGA are compared with those of ACO. It can be seen from the table that all the forces have been greatly reduced, in which the partial forces in the $x$ direction in plane II have been reduced to the single digits of $5.6 \mathrm{~N}$, and the reduction range has reached $-98.15 \%$. The total resultant force decreased by $-80.64 \%$. It can also be seen from the table that IGA can greatly improve the dynamic unbalance of the broken hammerhead shaft in theory.

Figure 7 shows the optimization results of each iteration of IGA, in which the black line represents the hammerheads optimization results of IGA curve, where each point of the curve is equal to the sum of the remaining four curves, and the red line represents the $x$ direction component force in plane I. The blue line represents the $y$ direction component force in plane $\mathrm{I}$, the orange line represents the $x$ direction component force in 
TABLe 6: Comparison of various statistical values by the applied algorithm.

\begin{tabular}{lcccc}
\hline Algorithms & Best & Mean & Worst & SD \\
\hline ACO & 762.9 & 845.9 & 954.7 & 62.0776 \\
GA & 402.3 & 541.7 & 643.6 & 63.5123 \\
PSO & 309.7 & 449.5 & 607.6 & 73.1067 \\
IGA & 147.7 & 161.7 & 190.3 & 13.5338 \\
\hline
\end{tabular}

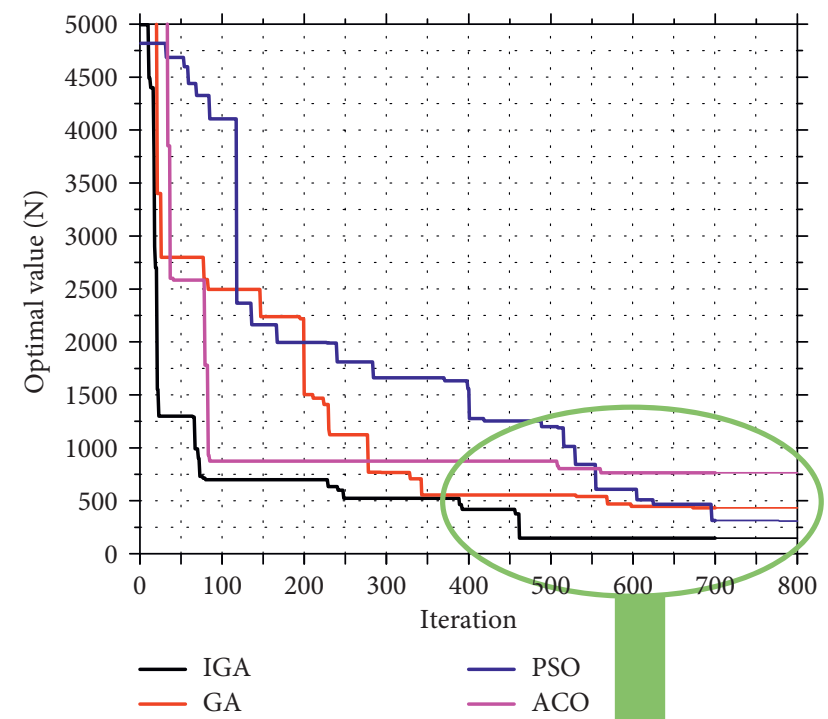

(a)

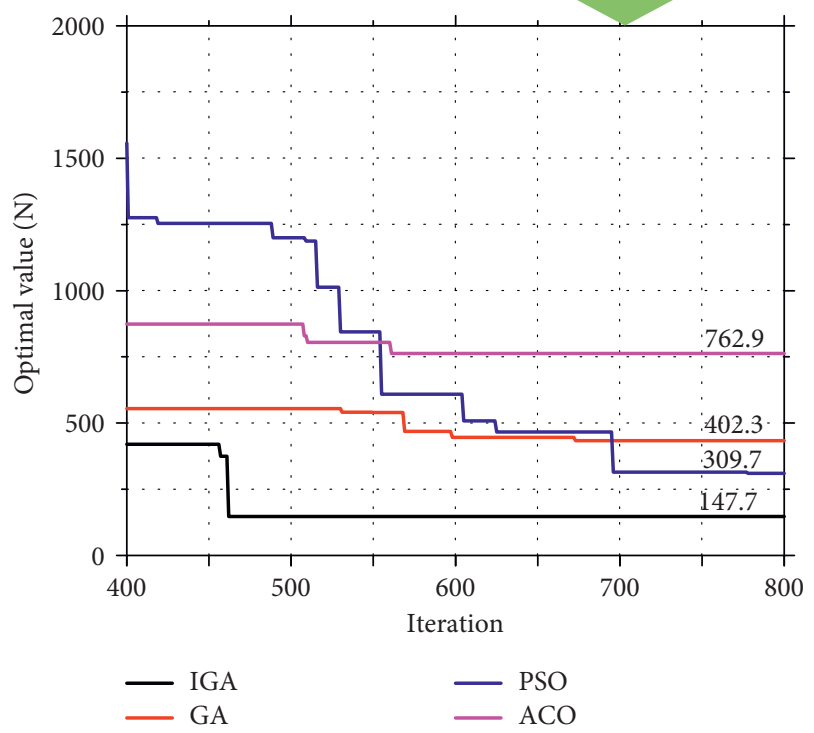

(b)

FIgURE 6: Result comparison of five consecutive calculations.

plane II, and the green line represents $y$ direction component force in plane I. Figure 8 shows the comparison of the two algorithms (IGA and ACO), where ACO is the result of the previous paper's algorithm running well once

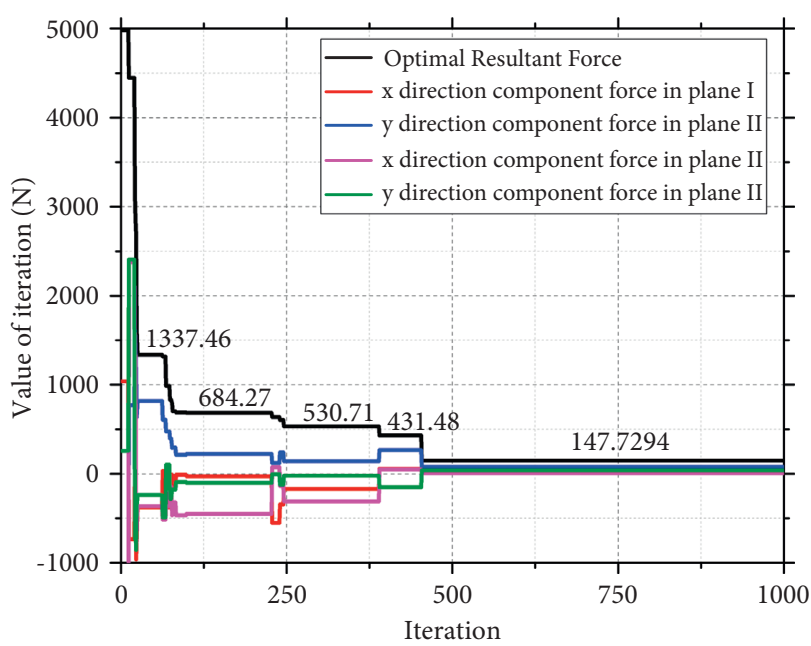

Figure 7: Optimization results of IGA.

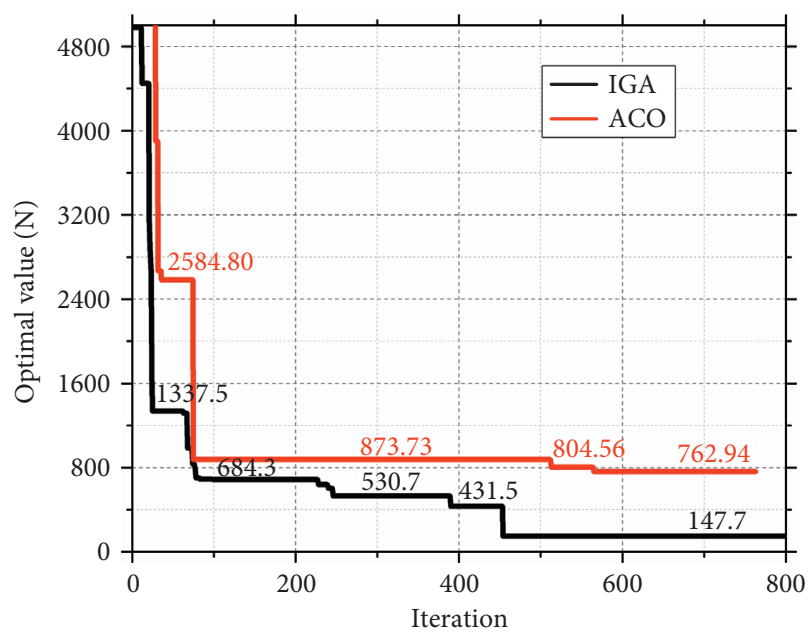

Figure 8: Comparison between IGA and ACO.

[10]. It can be seen from Figures 7 and 8 that the algorithm is convergent after 450 iterations. ACO basically converges after 80 iterations, IGA falls into the local optimal solution in 150-450 iterations, and SA of IGA is used to find the local optimal solution to obtain a better result. The results show that the resultant force of the shredder central shaft is reduced by $80.64 \%$. IGA has better convergence and robustness than ACO.

In Figures 9 and 10, the simulation results of IGA and ACO are shown, where the simulation time and the steps are $5.0 \mathrm{~s}$ and $0.01 \mathrm{~s}$, respectively. Initially, the rotor is unbalanced, and the hammerheads swing up incompletely. Then, the hammerheads run to the balance stage, and the curves tend to be normal. Figure 11 shows the comparison of simulation results in ADAMS to distinguish the difference of optimization curves between IGA and ACO on plane I and plane II. It can be seen from Figure 11 that the resultant force 


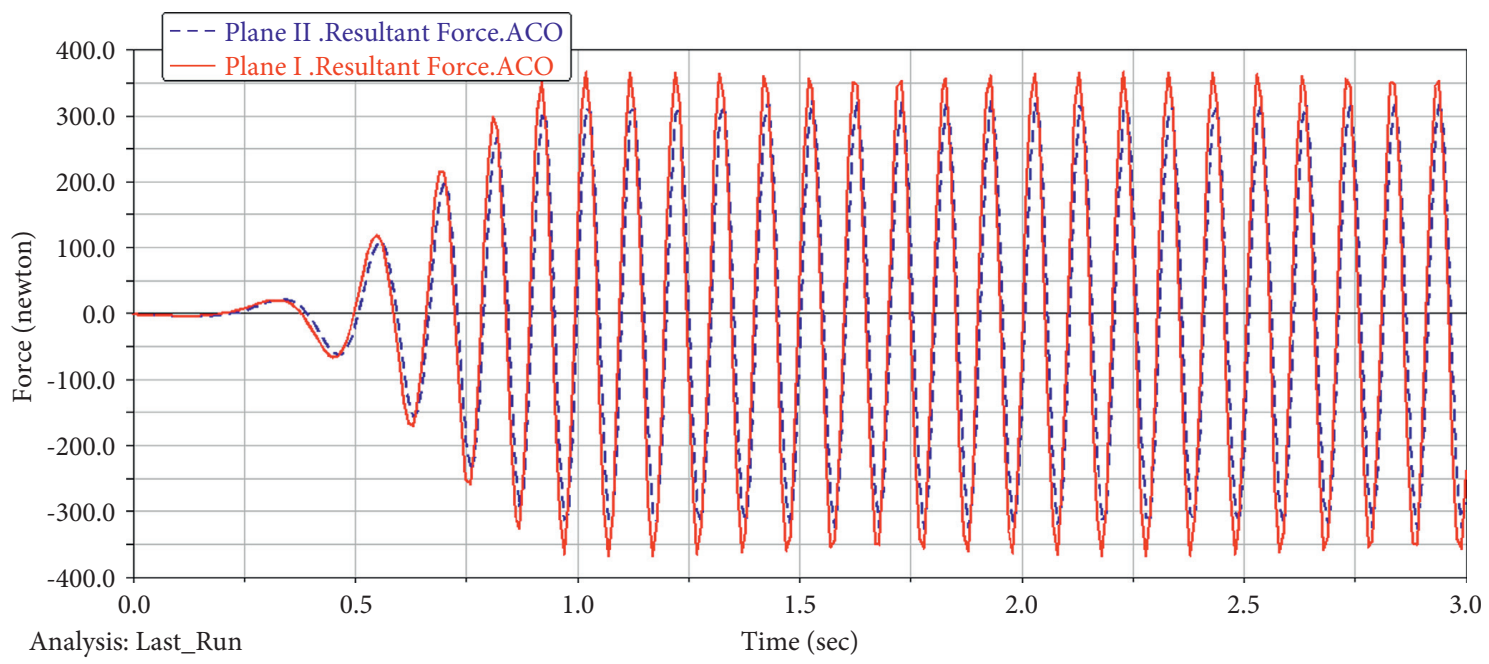

FIGURE 9: Simulation results of ACO in ADAMS.

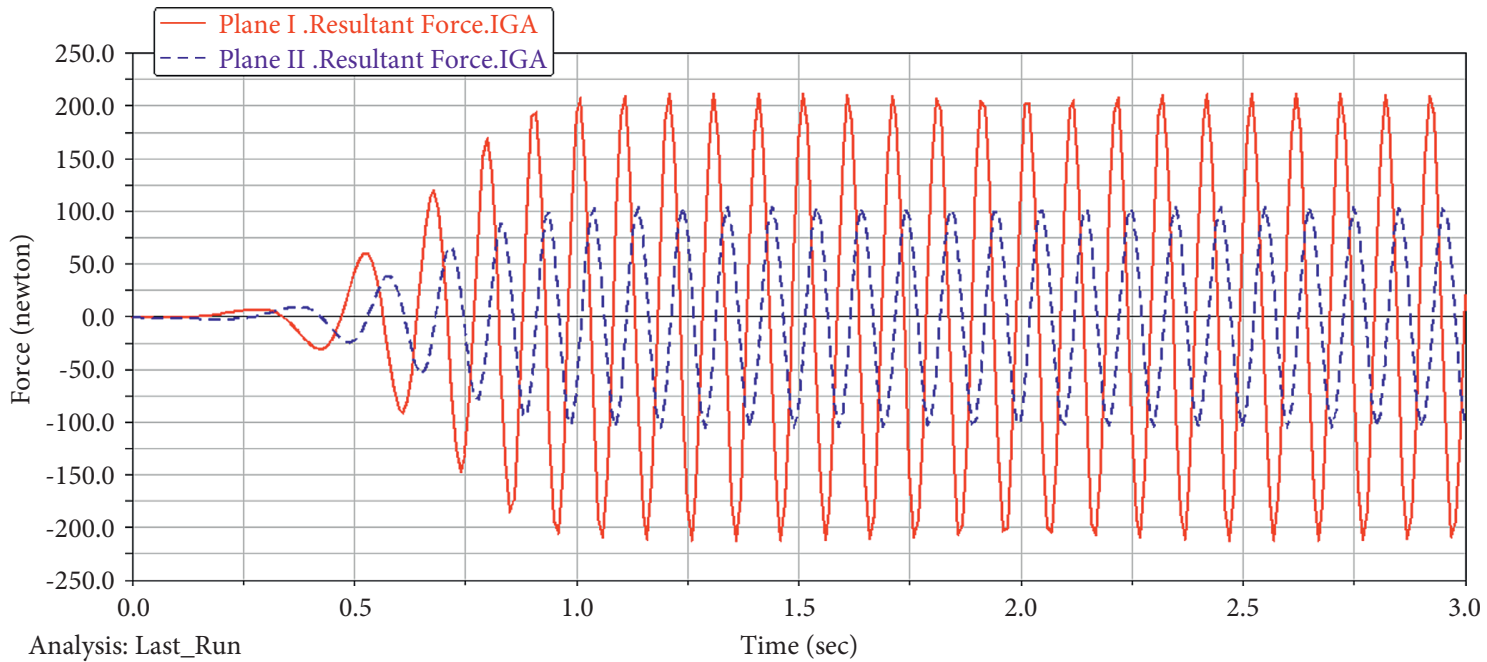

FIGURE 10: Simulation results of IGA in ADAMS.

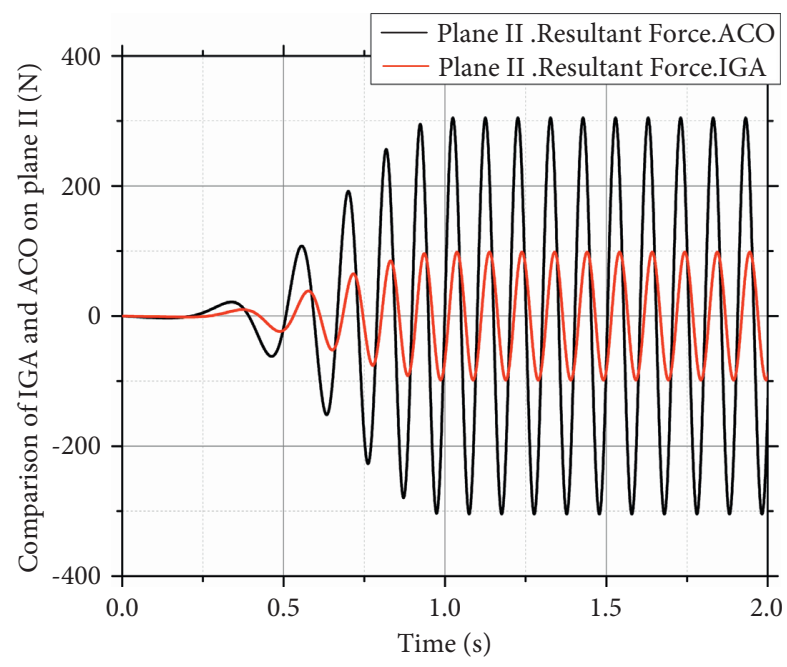

(a)

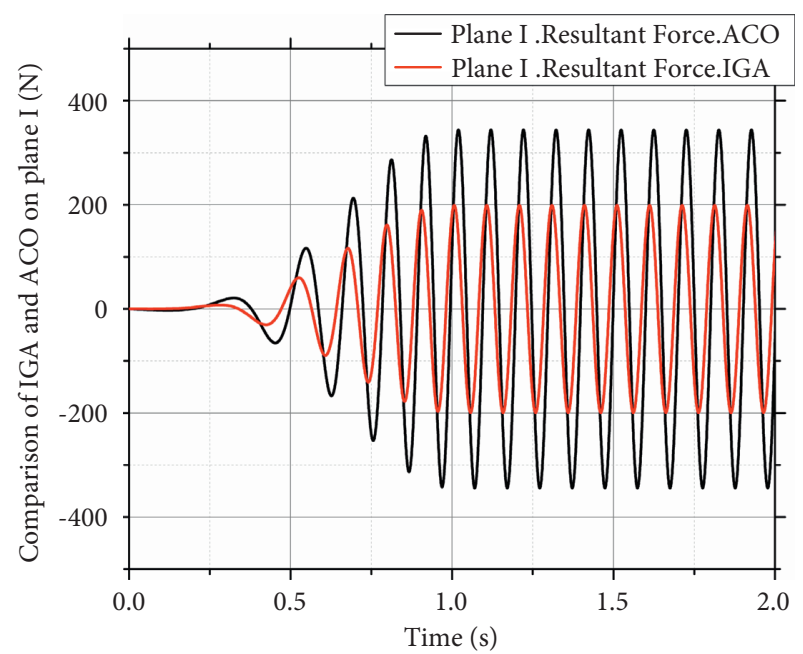

(b)

FIgURe 11: Comparison of simulation results between IGA and ACO in ADAMS. (a) Comparison of IGA and ACO on plane I. (b) Comparison of IGA and ACO on plane II. 


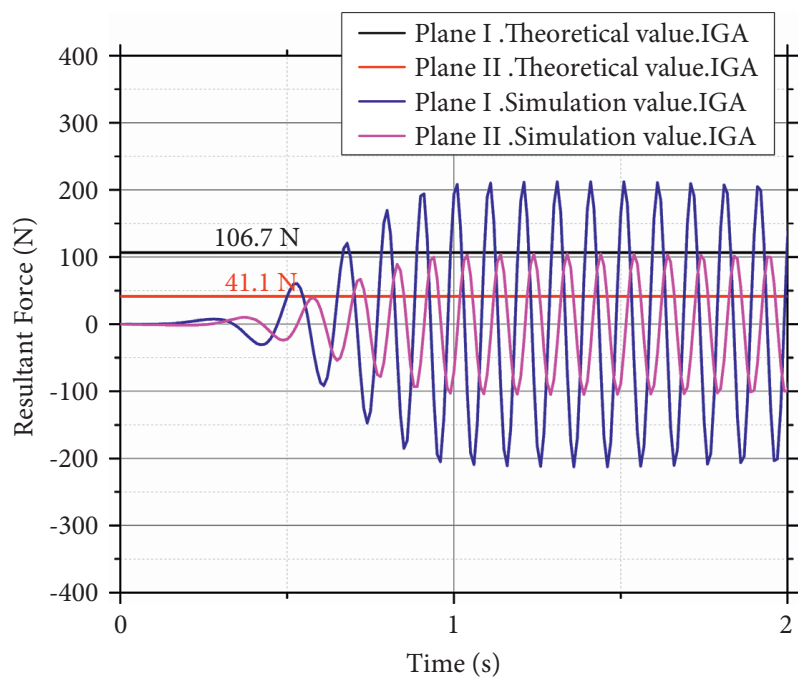

FIGURE 12: Comparison of IGA optimization results between theoretical and simulation values.

of IGA is significantly less than that of ACO on both plane I and plane II.

To illustrate the feasibility of the results of theoretical optimization, the comparison of IGA optimization results between theoretical and simulation values is shown in Figure 12. From this, it can be seen that the theoretical values of IGA (blue line and red line) are lower than the simulation values (black line and pink line). This is because that the quality of other components on the shredder is considered in the ADAMS simulation but not in the algorithm simulation. Obviously, the dynamic balance design achieves good performance and greatly reduces the cost of balance testing.

\section{Conclusions}

In this paper, a mathematical optimization model based on the principle of dynamic balance is established with the hammerhead arrangement of PFY2026 SMS as the research object, which is an MTSP problem. Based on the good characteristics of GA in global optimization and the good performance of SA at local optimal solutions, an improved genetic algorithm is proposed, which is obviously superior to ACO. The optimal resultant force is reduced by $80.64 \%$, and the dynamic unbalance on the shredder is greatly improved.

After optimization of IGA, the reduction degree of equal force in plane I and plane II is more than $60 \%$, the component force of $x$ direction in plane I can even be optimized to $5.6 \mathrm{~N}$, and the total resultant force of the two planes reaches $147.7 \mathrm{~N}$, compared to the result of ACO of about $762.94 \mathrm{~N}$. In this paper, the reasonable arrangement of hammerheads and fenders is put forward, which provides guidance for the optimization of the hammerhead arrangement of SMS.

ACO is specially proposed to solve the TSP problem. The IGA proposed in this paper is better than ACO in the hammerhead arrangement of SMS. In order to improve the convergence of the algorithm, a selection strategy based on fitness is adopted in this paper. The result analysis and
ADAMS simulation show that IGA is of great practical value in the optimization of hammerhead arrangement and can effectively guide the actual production.

\section{Abbreviations}

$\boldsymbol{A}_{i-1, i}: \quad$ Rotation matrix of coordinate system $o_{i}-x_{i} y_{i} z_{i}$ relative to $o_{i-1}-x_{i-1} y_{i-1} z_{i-1}$

$D_{i-1}: \quad$ Position vector of coordinate system $o_{i}-x_{i} y_{i} z_{i}$ relative to coordinate system $o_{i-1}-x_{i-1} y_{i-1} z_{i-1}$

$F_{i}: \quad$ Centrifugal force of $i$-th hammerhead $(\mathrm{N})$

$F_{\mathrm{I}}: \quad$ Total resultant force in plane I (N)

$F_{\text {II }}: \quad$ Total resultant force in plane II $(\mathrm{N})$

$f: \quad$ Actual deflection of central shaft $(\mathrm{m})$

$[f]: \quad$ Allowable deflection of central shaft $(m)$

$K: \quad$ Boltzmann constant $(\mathrm{J} / \mathrm{K})$

$m_{\mathrm{IAC}}: \quad$ Mass of hammerheads at all A positions of 1-11 planes $(\mathrm{kg})$

$m_{\mathrm{IAF}}$ : Mass of the common fenders at all A positions of 1-11 planes decomposed to plane $\mathrm{I}(\mathrm{kg})$

$m_{\text {IIAC: }} \quad$ Mass of hammerheads in all positions $\mathrm{A}$ of 1-11 planes to plane II $(\mathrm{kg})$

$m_{\mathrm{IIAF}}$ : Mass of the common fenders at all A positions of 1-11 planes on plane II $(\mathrm{kg})$

popsize: Total population size of IGA

$P_{c}: \quad$ Crossover probability rate

$P_{m}: \quad$ Mutation probability rate

$p^{T}{ }_{i j}: \quad$ Probability of the $i$-th individual of IGA

$q_{i}(x)$ : Fitness function value corresponding to the $i$-th individual of IGA

$r_{C}: \quad$ Diameter of the center of mass of hammerheads (m)

$r_{F}$ : Diameter of the center of mass of the common fenders $(\mathrm{m})$

$T_{0}: \quad$ Initial temperature $\left({ }^{\circ}\right)$

$T_{f}: \quad$ Termination temperature $\left({ }^{\circ}\right)$

$W: \quad$ Torsional cross section coefficient $\left(\mathrm{m}^{3}\right)$

$\alpha: \quad$ Temperature attenuation coefficient

$\omega: \quad$ Angular velocity of crusher rotor ( $\mathrm{rad} / \mathrm{s})$

$\sigma: \quad$ Actual shear stress of central shaft (MPa)

$[\sigma]: \quad$ Allowable shear stress of central shaft $(\mathrm{MPa})$.

\section{Data Availability}

Data supporting this research article are available from the corresponding author upon request.

\section{Conflicts of Interest}

The authors declare that they have no conflicts of interest.

\section{Acknowledgments}

This work was supported by the National Natural Science Foundation of China "Electromechanical coupling dynamics and adaptive control of multi-crawler travelling gears" (Grant no. 51775225) and the Graduate Innovation Fund of Jilin University (Grant no. 101832020CX111). 


\section{References}

[1] A Champasak, N Panagant, N Pholdee, S Bureerat, and A. R Yildiz, "Self-adaptive many-objective meta-heuristic based on decomposition for many-objective conceptual design of a fixed wing unmanned aerial vehicle," Aerospace Science and Technology, vol. 100, 2020.

[2] C. M. Aye, N. Pholdee, A. R. Yildiz, S. Bureerat, and S. M. Sait, "Multi-surrogate-assisted metaheuristics for crashworthiness optimisation," International Journal of Vehicle Design, vol. 80, no. 2/3/4, pp. 223-240, 2019.

[3] R. Sarangkum, K. Wansasueb, N. Panagant et al., "Automated design of aircraft fuselage stiffeners using multiobjective evolutionary optimisation," International Journal of Vehicle Design, vol. 80, no. 2/3/4, pp. 162-175, 2019.

[4] B. S. Yildiz, N. Pholdee, S. Bureerat, A. R. Yildiz, and S. M. Sait, "Sine-cosine optimization algorithm for the conceptual design of automobile components," Materials Testing, vol. 62, pp. 744-748, 2020.

[5] J. L. Ryan, T. G. Bailey, J. T. Moore, and W. B. Carlton, "Reactive tabu search in unmanned aerial reconnaissance simulations," in Proceedings of the Winter Simulation Conference, pp. 873-879, Washington, DC, USA, December 1998.

[6] Y.-B. Park, "A hybrid genetic algorithm for the vehicle scheduling problem with due times and time deadlines," International Journal of Production Economics, vol. 73, no. 2, pp. 175-188, 2001.

[7] T. Bektas, "The multiple traveling salesman problem: an overview of formulations and solution procedures," Omega, vol. 34, no. 3, pp. 209-219, 2006.

[8] T.-P. Dao and S.-C. Huang, "Design and multi-objective optimization for a broad self-amplified 2-DOF monolithic mechanism," Sädhanā, vol. 42, no. 9, pp. 1527-1542, 2017.

[9] T.-P. Dao, "Multiresponse optimization of a compliant guiding mechanism using hybrid taguchi-grey based fuzzy logic approach," Mathematical Problems in Engineering, vol. 2016, no. 6, 17 pages, Article ID 5386893, 2016.

[10] N. T. Tran, N. L. Chau, and T. P. Dao, "An effective hybrid approach of desirability, fuzzy logic, ANFIS and LAPO algorithm for optimizing compliant mechanism," Engineering with Computers, vol. 37, no. 4, 2020.

[11] W. Liu, S. Li, F. Zhao, and A. Zheng, "An ant colony optimization algorithm for the multiple traveling salesmen problem," in Proceedings of the 2009 4th IEEE conference on industrial electronics and applications, pp. 1533-1537, IEEE, Xian, China, May 2009.

[12] G. Wang, K. Zhao, X. Li, L. Li, and J. Wang, "Arrangement optimization of hammers and fenders on Scrap Metal Shredder using ant colony algorithms," Journal of Vibration and Control, vol. 18, no. 5, pp. 659-670, 2012.

[13] Z. W. Bo, L. Z. Hua, and Z. G. Yu, "Optimization of process route by genetic algorithms," Robotics and Computer-Integrated Manufacturing, vol. 22, no. 2, pp. 180-188, 2006.

[14] F. Cus and J. Balic, "Optimization of cutting process by GA approach," Robotics and Computer-Integrated Manufacturing, vol. 19, no. 1, pp. 113-121, 2003.

[15] J. Holland, "Adaptation in natural and artificial systems: an introductory analysis with applications to biology, control and artificial intelligence," Ann Arbor, vol. 6, no. 2, pp. 126-137, 1992, bknumber $=6267401$.

[16] S. S. F. Smith and G. C. Smith, "An enhanced genetic algorithm for automated assembly planning," Robotics and Computer-Integrated Manufacturing, vol. 18, no. 5, pp. 355-364, 2002.
[17] A. E. Carter and C. T. Ragsdale, "A new approach to solving the multiple traveling salesperson problem using genetic algorithms," European Journal of Operational Research, vol. 175, no. 1, pp. 246-257, 2006.

[18] N. S. Kwak and J. Lee, "An enhancement of selection and crossover operations in real-coded genetic algorithm for large-dimensionality optimization," Journal of Mechanical Science and Technology, vol. 30, no. 1, pp. 237-247, 2016.

[19] J. R. Cho and J. H. Lee, "Multi-objective optimum design of TBR tire structure for enhancing the durability using genetic algorithm," Journal of Mechanical Science and Technology, vol. 31, no. 12, pp. 5961-5969, 2017.

[20] Z Meng, G Li, X Wang, S. M Sait, and A. R Yildiz, “A comparative study of metaheuristic algorithms for reliabilitybased design optimization problems," Archives of Computational Methods in Engineering, vol. 28, no. 4, pp. 1853-1869, 2020.

[21] S. Gupta, H. Abderazek, and B. S. Yıldiz, "Comparison of metaheuristic optimization algorithms for solving constrained mechanical design optimization problems," Expert Systems with Applications, vol. 183, Article ID 115351, 2021.

[22] S. Kirkpatrick, C. D. Gelatt, and M. P. Vecchi, "Optimization by simulated annealing," Science, vol. 220, pp. 671-680, 1983.

[23] J. Lee and G. E. Johnson, "Optimal tolerance allotment using a genetic algorithm and truncated Monte Carlo simulation," Computer-Aided Design, vol. 25, pp. 601-611, 1983.

[24] Z. G. Wang, Y. S. Wong, and M. Rahman, "Optimisation of multi-pass milling using genetic algorithm and genetic simulated annealing," International Journal of Advanced Manufacturing Technology, vol. 24, no. 9-10, pp. 727-732, 2004.

[25] W. Jwo, C. Liu, and C. Liu, "Large-scale optimal VAR planning by hybrid simulated annealing/genetic algorithm," International Journal of Electrical Power \& Energy Systems, vol. 21, no. 1, pp. 39-44, 1999.

[26] X. Cai, H. Zhao, S. Shang et al., "An improved quantuminspired cooperative co-evolution algorithm with mulistrategy and its application," Expert Systems Applications, vol. 171, Article ID 114629, 2021.

[27] W. Deng and J. Xu, "An enhanced MSIQDE algorithm with novel multiple strategies for global optimization problems," IEEE Transactions on Systems, Man, and Cybernetics: Systems, vol. 50, 2020.

[28] D. Wu, S. Shang, C. Xing et al., "Quantum differential evolution with cooperative coevolution framework and hybrid mutation strategy for large scale optimization," KnowledgeBased Systems, vol. 224, 2021.

[29] J. H. Holland, Adaptation in Natural and Artificial Systems, pp. 126-137, MIT Press, Cambridge, MA, USA, 1992.

[30] W. M. Spears and K. A. De Jong, "An Analysis of Multi-Point Crossover," in Foundations of Genetic Algorithms, G. Rawlins, Ed., pp. 301-315, 1991.

[31] P. Tang and M. Tseng, "Adaptive directed mutation for realcoded genetic algorithms," Applied Soft Computing, vol. 13, no. 1, pp. 600-614, 2013.

[32] K. Deb and J. Sundar, "Reference point based multi-objective optimization using evolutionary algorithms," in Proceedings of the 8th annual conference on Genetic and evolutionary computation, pp. 635-642, Seattle, WA, USA, July 2006.

[33] H. P. Schwefel, Numerical Optimization of Computer Models, John Wiley \& Sons, Hoboken, NJ, USA, 1981.

[34] K. Deb and M. Goyal, "A combined genetic adaptive search (GeneAS) for engineering design," Computer Science and Informatics, vol. 26, pp. 30-45, 1996. 
[35] Z. Michalewicz, Genetic Algorithms + Data Structures= Evolution Programs, Springer Science \& Business Media, 2013.

[36] H. G. Beyer and H. P. Schwefel, "Evolution strategies-A comprehensive introduction," Natural Computing, vol. 1, no. 1, pp. 3-52, 2002.

[37] P. M. Mateo and I. Alberto, "A mutation operator based on a Pareto ranking for multi-objective evolutionary algorithms," Journal of Heuristics, vol. 18, no. 1, pp. 53-89, 2012.

[38] Z. G. Wang, M. Rahman, Y. S. Wong, and J. Sun, "Optimization of multi-pass milling using parallel genetic algorithm and parallel genetic simulated annealing," International Journal of Machine Tools and Manufacture, vol. 45, no. 15, pp. 1726-1734, 2005.

[39] T. Kellegöz, B. Toklu, and J. Wilson, "Comparing efficiencies of genetic crossover operators for one machine total weighted tardiness problem," Applied Mathematics and Computation, vol. 199, no. 2, pp. 590-598, 2008. 